\title{
Searching for the Transit of the Earth-mass Exoplanet Proxima Centauri b in Antarctica: Preliminary Result
}

\author{
Hui-Gen Liu ${ }^{1,2}$ (1) , Peng Jiang ${ }^{3,4}$ (1) , Xingxing Huang ${ }^{4,5}$, Zhou-Yi Yu ${ }^{1,2}$, Ming Yang ${ }^{1,2}$, Minghao Jia ${ }^{6,7}$, \\ Supachai Awiphan ${ }^{8}$, Xiang Pan ${ }^{3,4,5}$, Bo Liu ${ }^{3,4,5}$, Hongfei Zhang ${ }^{6,7}$, Jian Wang ${ }^{6,7}$, Zhengyang Li $^{9}$, Fujia Du ${ }^{9}$,

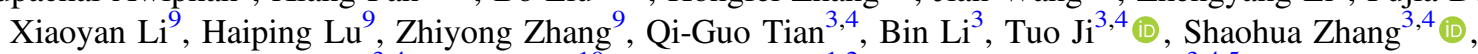 \\ Xiheng Shi ${ }^{3,4}$ (10), Ji Wang ${ }^{10}$ (1) , Ji-Lin Zhou ${ }^{1,2}$ (1) , and Hongyan Zhou ${ }^{3,4,5}$ (1) \\ ${ }^{1}$ School of Astronomy and Space Science, Nanjing University, Nanjing, Jiangsu, 210093, China; huigen@nju.edu.cn \\ ${ }^{2}$ Key Laboratory of Modern Astronomy and Astrophysics (Nanjing University), Ministry of Education, Nanjing, Jiangsu, 210093, China \\ ${ }^{3}$ Polar Research Institute of China, Jinqiao Rd. 451, Shanghai, 200136, China; jpaty @ mail.ustc.edu.cn \\ ${ }^{4}$ Key Laboratory for Research in Galaxies and Cosmology, The University of Science and \\ Technology of China, Chinese Academy of Sciences, Hefei, Anhui, 230026, China \\ ${ }^{5}$ Department of Astronomy, The University of Science and Technology of China, Hefei, Anhui, 230026, China \\ ${ }^{6}$ Department of Modern Physics, The University of Science and Technology of China, Hefei, Anhui, 230026, China \\ State Key Laboratory of Technologies of Particle Detection and Electronics, Department of Modern Physics, \\ University of Science and Technology of China, Hefei, Anhui, 230026, China \\ ${ }^{8}$ National Astronomical Research Institute of Thailand, 191 Huay Kaew Road, Amphoe Mueang, Chiang Mai, 50200, Thailand \\ ${ }^{9}$ National Astronomical Observatories/Nanjing Institute of Astronomical Optics and Technology, \\ Chinese Academy of Sciences, Nanjing, Jiangsu, 210042, China \\ ${ }^{10}$ Department of Astronomy, California Institute of Technology, MC 249-17, 1200 E. California Boulevard, Pasadena, CA 91106, USA \\ Received 2017 March 6; revised 2017 November 14; accepted 2017 November 14; published 2017 December 13
}

\begin{abstract}
Proxima Centauri is known as the closest star to the Sun. Recently, radial velocity (RV) observations revealed the existence of an Earth-mass planet around it. With an orbital period of $\sim 11$ days, Proxima Centauri b is probably in the habitable zone of its host star. We undertook a photometric monitoring campaign to search for its transit, using the Bright Star Survey Telescope at the Zhongshan Station in Antarctica. A transit-like signal appearing on 2016 September 8 has been tentatively identified. Its midtime, $T_{C}=2,457,640.1990 \pm 0.0017 \mathrm{HJD}$, is consistent with the predicted ephemeris based on the RV orbit in a $1 \sigma$ confidence interval. Time-correlated noise is pronounced in the light curve of Proxima Centauri, affecting the detection of transits. We develop a technique, in a Gaussian process framework, to gauge the statistical significance of a potential transit detection. The tentative transit signal reported here has a confidence level of $2.5 \sigma$. Further detection of its periodic signals is necessary to confirm the planetary transit of Proxima Centauri b. We plan to monitor Proxima Centauri in the next polar night at Dome A in Antarctica, taking advantage of continuous darkness. Kipping et al. reported two tentative transit-like signals of Proxima Centauri b observed by the Microvariability and Oscillation of Stars space telescope in 2014 and 2015. The midtransit time of our detection is 138 minutes later than that predicted by their transit ephemeris. If all of the signals are real transits, the misalignment of the epochs plausibly suggests transit timing variations of Proxima Centauri b induced by an outer planet in this system.
\end{abstract}

Key words: methods: data analysis - planets and satellites: terrestrial planets - stars: individual (Proxima Centauri) - techniques: photometric

Supporting material: machine-readable table

\section{Introduction}

The detection of terrestrial exoplanets is important for studying the population, diversity, and habitability of planets beyond the solar system. Limited by atmospheric scintillation (Dravins et al. 1998), ground-based telescopes cannot deliver a photometric precision comparable to space-based telescopes, which is required to detect transits of terrestrial planets around solar-like stars. Red dwarfs have much smaller size than the Sun, i.e., $R \leqslant 0.15 R_{\odot}$. The transits of Earth-size planets around red dwarfs, with a typical depth of $\sim 0.5 \%$, are considerable for observation using ground-based telescopes (e.g., Charbonneau et al. 2009; Gillon et al. 2016, 2017). Aside from their small sizes, the masses of red dwarf planet hosts are small. Periodic radial velocity (RV) modulations of red dwarfs induced by their terrestrial planets are observable using current spectrographic technique with a precision of $\sim 1 \mathrm{~m} \mathrm{~s}^{-1}$ (Bouchy et al. 2001; Pepe et al. 2011; Fischer et al. 2016). Moreover, red dwarfs are abundant near the Sun. The survey conducted by the REsearch
Consortium On Nearby Stars (RECONS; Henry et al. 2006) shows that $\sim 69 \%$ of stars within $10 \mathrm{pc}$ are red dwarfs.

Proxima Centauri, a red dwarf, is well-known as the nearest star to the Sun, with a distance of $1.3008 \pm 0.0006 \mathrm{pc}$ (Kervella et al. 2016). Proxima Centauri and $\alpha$ Centauri A and B are in a hierarchical triple star system. $\alpha$ Centauri A and B constitute a binary subsystem, while Proxima Centauri is currently close to the apastron $\left(13.0_{-0.1}^{+0.3} \mathrm{kAU}\right)$ of its orbit $\left(e=0.5_{-0.09}^{+0.08}\right)$ around $\alpha$ Centauri $\mathrm{AB}$ (Kervella et al. 2017). Planets around these three stars, if discovered, would plausibly be the closest exoplanets to the Earth.

First, an Earth-mass planet, $\alpha$ Centauri Bb, was claimed by Dumusque et al. (2012) based on RV measurements using the High Accuracy Radial velocity Planet Searcher (HARPS). However, the weak planetary signal with a semi-amplitude of $\sim 0.5 \mathrm{~m} \mathrm{~s}^{-1}$ is most likely spurious, arising from the time sampling of the original data and intrinsic stellar activity (Rajpaul et al. 2015, 2016). Analyzing the light curve observed 
using the Hubble Space Telescope (HST) over $40 \mathrm{hr}$, Demory et al. (2015) detected a single transit-like event but it is unlikely associated with $\alpha$ Centauri Bb. Recently, an Earth-mass planet around Proxima Centauri was discovered, using two highprecision RV instruments (Anglada-Escudé et al. 2016), HARPS and the Ultraviolet and Visual Echelle Spectrograph (UVES), jointly. With an orbital period of $11.186_{-0.002}^{+0.001}$ days, the surface of Proxima Centauri b is probably temperate and habitable.

The geometric probability of transit for Proxima Centauri b is just about $1.5 \%$. Nevertheless, efforts to detect its planetary transit are valuable, as such a discovery would be very important. The planetary mass and radius can be accurately measured, enabling us to study its structure and internal compositions (Fortney et al. 2007; Dressing et al. 2015). Furthermore, its atmosphere can be characterized using transmission spectroscopy technique. The habitability of a planet is very sensitive to the properties and compositions of its atmosphere (Kreidberg \& Loeb 2016; Meadows et al. 2016; Ribas et al. 2016; Turbet et al. 2016). The transit depth of Proxima Centauri b would be $\sim 5 \mathrm{mmag}$, assuming it has a similar density to the Earth (Anglada-Escudé et al. 2016). The required photometric precision can be easily achieved with small aperture telescopes for bright stars, and thus searching for the planetary transit of Proxima Centauri $b$ is affordable.

The first attempt was made by Kipping et al. (2017) using archival data of the Microvariability and Oscillation of Stars (MOST) space telescope observed in 2014 (for 12.5 days) and in 2015 (for 31 days). Proxima Centauri was observed for a fraction $(<50 \%)$ of each MOST 101 minute orbit when it was observable, with an average sampling rate of about 1 minute. A candidate transit signal with two events were detected marginally. However, the signal cannot be recovered in the light curve observed by the HATSouth telescope network (Bakos et al. 2013). Proxima Centauri is a moderately active star and has a rotation period of $\sim 83$ days (Christian et al. 2004; Kiraga \& Stepien 2007; Davenport et al. 2016). Time-correlated structure and long-term trends are pronounced in its light curve (Kipping et al. 2017). Considering that the expected transit duration of Proxima Centauri b is about $1 \mathrm{hr}$, the MOST light curve, with discrete sampling windows with width of $<50$ minutes but separated by $>50$ minutes, is not optimal for disentangling the potential transit signals from time-correlated noise. High-cadence consecutive photometric monitoring are valuable for Proxima Centauri.

We started the photometric monitoring of Proxima Centauri in Antarctica right after the announcement of the discovery of Proxima Centauri b (Anglada-Escudé et al. 2016). In August and September, Proxima Centauri is not observable at almost all observatories around the world, except for the sites in Antarctica. Our observations were carried out using the Bright Star Survey Telescope (BSST; Tian et al. 2016) deployed at the Chinese Antarctic Zhongshan station (south 69 $22^{\prime} 23^{\prime \prime}$, east $\left.76^{\circ} 22^{\prime} 17^{\prime \prime}\right)$. In this paper, the BSST telescope is briefly introduced in Section 2. Observation and data reduction are presented in Section 3. We search for transit signals in the BSST light curves in Section 4. A discussion and brief summary are presented in Section 5 and Section 6, respectively.

\section{The Bright Star Survey Telescope in Antarctica}

Dome A, on the top of the ice cap in Antarctica, is one of the most promising astronomical sites on Earth (Yang et al. 2009;
Bonner et al. 2010; Shi et al. 2016). The Chinese Antarctic Kunlun station (south $80^{\circ} 25^{\prime} 01^{\prime \prime}$, east $77^{\circ} 06^{\prime} 58^{\prime \prime}$ ), which is $7.3 \mathrm{~km}$ away from Dome A in the southwest, is currently hosting two wide-field telescopes with aperture of $500 \mathrm{~mm}$ (the Antarctic Survey Telescopes; Yuan et al. 2008) and will be developed to support more astronomical facilities in the near future. The survey of transiting extrasolar planets, taking advantage of the continuous darkness and large clear-sky fraction (>90\%; Zou et al. 2010; Law et al. 2013; Wang et al. 2014) in the winter months, is one of the main scientific goals at Kunlun station.

BSST (Tian et al. 2016) is optimally designed for searching planetary transits of bright stars and will join the ongoing survey at Kunlun station. It has an aperture size of $300 \mathrm{~mm}$ and is equipped with a large frame $4 \mathrm{~K} \times 4 \mathrm{~K} \mathrm{CCD}$ camera to receive starlight from a $3^{\circ} .4 \times 3^{\circ} .4$ field of view $(\mathrm{Li}$ et al. 2015). An autonomous observation and control system developed on the basis of EPICS ${ }^{11}$ and RTS2 (Kubánek et al. 2006, 2008) enables BSST to run robotically (Zhang et al. 2016). Low-temperature-resistant testing in the laboratory demonstrated that the telescope can work functionally in ambient temperatures down to $-70^{\circ} \mathrm{C}$ (Tian et al. 2016). In 2016 March, BSST was installed at the Chinese Antarctic Zhongshan station, on Larsemann Hills in Prydz Bay, for testing operation. Its first observation season in Antarctica ceased at the end of 2016 September. We plan to move BSST to Kunlun Station in two years.

\section{Observation and Data Reduction}

We monitored the photometry of Proxima Centauri over 10 nights between 2016 August 29 and September 21. The star was observed in its white light, without any filter. The typical airmass toward Proxima Centauri was 1.4 all throughout the observation campaign. Five exposures of $3 \mathrm{~s}$ are combined as a photometric point, and a typical cadence of data points is $220 \mathrm{~s}$.

Raw images are first corrected for bias and flat-field using standard procedures. We manually select 62 nearby stars with similar brightness to Proxima Centauri for aperture photometry. Since the observed star field is quite dense, the background for each star can hardly be determined in a simple way using concentric circles. We then manually select the nearest region around each star without significant starlight to estimate its background level. Aperture photometry of Proxima Centauri and the 62 preliminary reference stars is extracted for each image. The five consecutive data points of each star are then averaged numerically to produce the raw light curves.

We reject unstable stars among the 62 preliminary reference stars by comparing their raw light curves mutually and iteratively. Finally, the six nearest stable stars around Proxima Centauri are selected as reference stars for differential photometry. We further identify the photometric points suffering from significant extinction due to clouds in the raw light curves. These points are then clipped to produce the clean light curves.

The differential light curve of Proxima Centauri is derived by comparing its clean light curve with the average clean light curve of the six reference stars. Among the six stable stars, we select a star, which has almost the same brightness as Proxima Centauri, to estimate the photometric precision

\footnotetext{
${ }^{11}$ Experimental Physics and Industrial Control System, http://www.aps.anl. gov/epics/.
} 
Table 1

Photometry of Proxima Centauri Observed by BSST

\begin{tabular}{lccc}
\hline \hline $\begin{array}{l}\text { HJD-2,457,545 } \\
\text { (day) }\end{array}$ & Flux $^{\mathrm{a}}$ & Flux $^{\mathrm{b}}$ & Uncertainty \\
\hline 85.1684 & 0.9956 & 0.9941 & 0.0049 \\
85.1709 & 0.9915 & 0.9889 & 0.0049 \\
85.1739 & 0.9969 & 0.9962 & 0.0049 \\
85.1766 & 0.9941 & 0.9926 & 0.0049 \\
85.1792 & 1.0053 & 1.0075 & 0.0049 \\
85.1817 & 1.0069 & 1.0098 & 0.0049 \\
85.1843 & 1.0070 & 1.0101 & 0.0049 \\
85.1871 & 1.0010 & 1.0023 & 0.0049 \\
85.1911 & 1.0081 & 1.0118 & 0.0049 \\
85.1940 & 0.9979 & 0.9986 & 0.0049 \\
85.1968 & 1.0015 & 1.0034 & 0.0049 \\
85.1995 & 1.0013 & 1.0032 & 0.0049 \\
85.2022 & 1.0008 & 1.0027 & 0.0049 \\
85.2053 & 1.0022 & 1.0046 & 0.0049 \\
85.2089 & 0.9954 & 0.9958 & 0.0049 \\
\hline
\end{tabular}

Notes. Time has been converted to HJD (Heliocentric Julian Day) from UTC (Universal Time Coordinated) time. This table is available in its entirety in machine-readable form in the online journal. A portion is shown here for guidance regarding its form and content.

${ }^{a}$ Normalized differential flux of Proxima Centauri.

${ }^{\mathrm{b}}$ Detrended flux by fitting a cubic polynomial to data observed in individual nights.

(This table is available in its entirety in machine-readable form.)

independently. The scatter of its differential light curve, which is measured by comparing the clean light curve of the selected reference star with the average clean light curve of the other five reference stars, is reported as the photometric precision for Proxima Centauri. Precision is estimated night by night. Overall, the average precision is $4.6 \mathrm{mmag}$, which is significantly larger than the theoretical photon noise of $\sim 1$ mmag. We estimate the amplitude of the atmospheric scintillation (Dravins et al. 1998) as

$$
\sigma=0.09 \frac{X^{3 / 2}}{D^{2 / 3} \sqrt{2 t}} \exp \left(-\frac{h}{8}\right)
$$

where $X=1.4$ is the airmass, $D=30$ the diameter of the telescope in $\mathrm{cm}, t=15$ the exposure time in s, and $h=0$ the altitude of observatory in $\mathrm{km}$, yielding $\sigma \approx 3 \mathrm{mmag}$. Thus, the photometric precision is mainly limited by the atmospheric scintillation and possible systematic errors.

The normalized light curve of Proxima Centauri is tabulated in Table 1 and illustrated in Figure 1. Time-correlated noise is pronounced in the BSST light curve. In order to enhance the visibility of the potential transit features, we detrend the light curve by fitting a cubic polynomial to the data observed in individual nights. That procedure removes the long-term variations, but can preserve the features with short timescales as well as potential transit signals. The detrended light curve of Proxima Centauri is tabulated in Table 1 as well. Note that the detrended light curve is only used for visual inspection of transit features, not for statistical analysis since time-correlated noise has been reduced artificially.

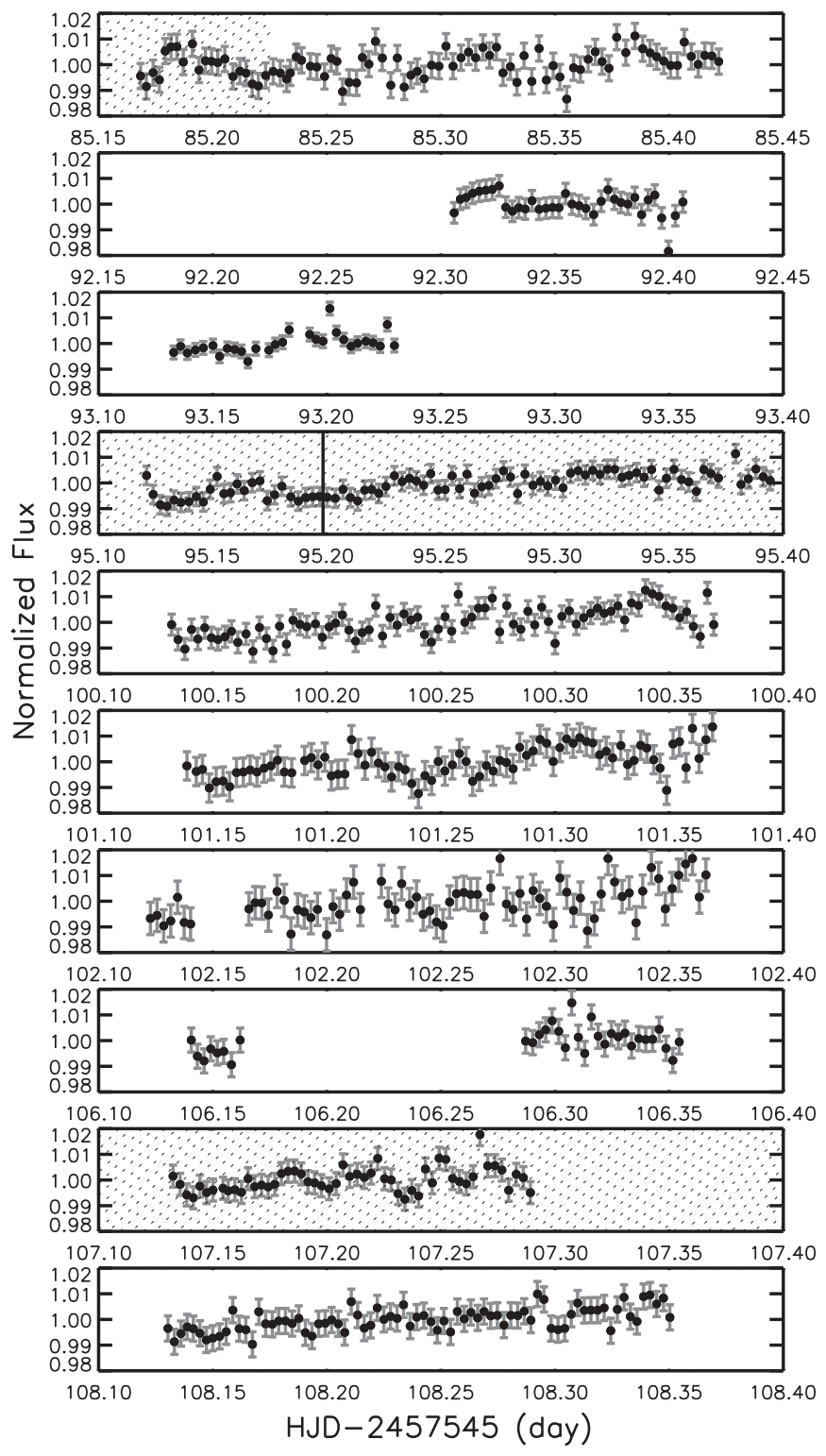

Figure 1. Normalized differential light curve of Proxima Centauri observed using BSST at the Antarctic Zhongshan station during 10 nights from 2016 August 29 to September 21. Time has been converted to HJD from UTC. The average photometry precision is $4.6 \mathrm{mmag}$. The shaded areas are time within $1 \sigma$ predicted transit windows $\left(T_{\mathrm{IC}}=2,457,629.56 \pm 0.67, T_{\mathrm{IC}}=2,457,640.74 \pm 0.67 \mathrm{HJD}\right.$, and $\left.T_{\mathrm{IC}}=2,457,651.93 \pm 0.67\right)$. The transit ephemeris is derived by Kipping et al. (2017) using the radial velocity solution of Anglada-Escudé et al. (2016). The vertical solid line indicates the midtransit time of the candidate event identified in Section 4.

\section{Searching for Transit Signal in BSST Data}

\subsection{A Candidate Transit Event}

If Proxima Centauri $b$ transits, the transit minimum would appear at the time of inferior conjunction. Using the RV solution of Anglada-Escudé et al. (2016), Kipping et al. (2017) derived the time of inferior conjunction $T_{\mathrm{IC}}=2,456,678.78 \pm 0.56 \mathrm{HJD}$ at an original epoch and the orbital period $P=11.1856 \pm$ 0.0013 days. We adopt the transit ephemeris in Kipping et al. (2017) to predict the time of transit window in the BSST light curve. The first observing night is partially covered by the $1 \sigma$ window of interest $\left(T_{\mathrm{IC}}=2,457,629.56 \pm 0.67 \mathrm{HJD}\right.$ for the 
85th epoch). The fourth and ninth observing nights are fully covered $\left(T_{\mathrm{IC}}=2,457,640.74 \pm 0.67\right.$ HJD for the 86th epoch, $T_{\text {IC }}=2,457,651.93 \pm 0.67 \mathrm{HJD}$ for the 87th epoch). The windows of interest for the transit are indicated by the shaded regions in Figure 1.

Besides the transit ephemeris, the transit duration and depth are requisite parameters to characterize the planetary transit signals in light curves. Proxima Centauri has a stellar mass of $M_{*}=0.120 \pm 0.015 M_{\odot}$ and a stellar radius of $R_{*}=$ $0.141 \pm 0.021 R_{\odot}$ (Delfosse et al. 2000; Boyajian et al. 2012). The minimum mass of Proxima Centauri $\mathrm{b}$ is $M_{p} \sin i=$ $1.27_{-0.17}^{+0.19} M_{\oplus}$ (Anglada-Escudé et al. 2016). Assuming a circular planetary orbit, the transit duration can be expressed as

$$
T_{d}=\frac{P}{\pi} \sin ^{-1}\left(\frac{\sqrt{\left(R_{*}+R_{p}\right)^{2}-\left(b R_{*}\right)^{2}}}{a}\right),
$$

where $b$ is the impact parameter, $R_{p}$ is the planetary radius, and $a \approx \sqrt[3]{\frac{G M_{*} T^{2}}{4 \pi^{2}}}=0.048$ au is the semimajor axis. If Proxima Centauri $\mathrm{b}$ transits, its true mass would be almost identical to the minimum mass $M_{p} \approx 1.27 M_{\oplus}$. Assuming that the planet has a density similar to the Earth, the planetary radius can therefore be estimated as $R_{p} \approx 1.08 R_{\oplus}$. Then, the planetary transit duration is about 75 minutes when $b=0$, and is about 66 minutes when $b=0.5$. The planetary transit depth is $D=\left(R_{p} / R_{*}\right)^{2}=0.48 \%$.

We inspect the detrended BSST light curve of Proxima Centauri to look for features similar to the predicted transit signal. A candidate transit event at the epoch of $\sim 2,457,640.2 \mathrm{HJD}$ is identified. It is in the $1 \sigma$ window of interest of $T_{\mathrm{IC}}=2,457,640.74 \pm 0.67 \mathrm{HJD}$ for the 86th epoch (see Figure 1). We fit the candidate event using a simple transit model assuming the star is a uniform source (Mandel \& Agol 2002). There are three free parameters in the model, i.e., the time of transit minimum $T_{\mathrm{C}}$, transit duration $T_{d}$, and transit depth $D$. The fit is carried out with the Levenberg-Marquardt least-squares algorithm, ${ }^{12}$ yielding $T_{\mathrm{C}}=2,457,640.1983 \pm$ $0.0020 \mathrm{HJD}, T_{d}=82 \pm 6$ minutes, and $D=5.5 \pm 1.1 \mathrm{mmag}$. The best-fitted transit parameters are compatible with the estimated parameters of the transit signal induced by Proxima Centauri $b$, considering the uncertainty of the stellar parameters and the parameters of the planetary orbit. The candidate transit event and its best-fitted model are presented in Figure 2.

\subsection{Time-correlated Noise and Statistical Significance}

Time-correlated noise, induced by changing atmospheric conditions, and instrumental and stellar variability, has a very important effect on the detection threshold of planetary transit surveys (Pont et al. 2006). Proxima Centauri exhibits a pronounced time-correlated structure in its light curve. Thus, it is necessary to take correlated noise into account when gauging the statistical significance of the candidate transit signal.

We estimate the time-correlated noise $\sigma_{\text {tc }}$ in the BSST normalized light curve, following the procedure introduced by Pont et al. (2006). We calculate the mean flux $F_{j}$ over a sliding interval of length of the transit duration obtained in Section 4.1.

\footnotetext{
$\overline{12}$ IDL procedures developed by Craig B. Markwardt, http://cow.physics. wisc.edu/ craigm/idl/idl.html.
}

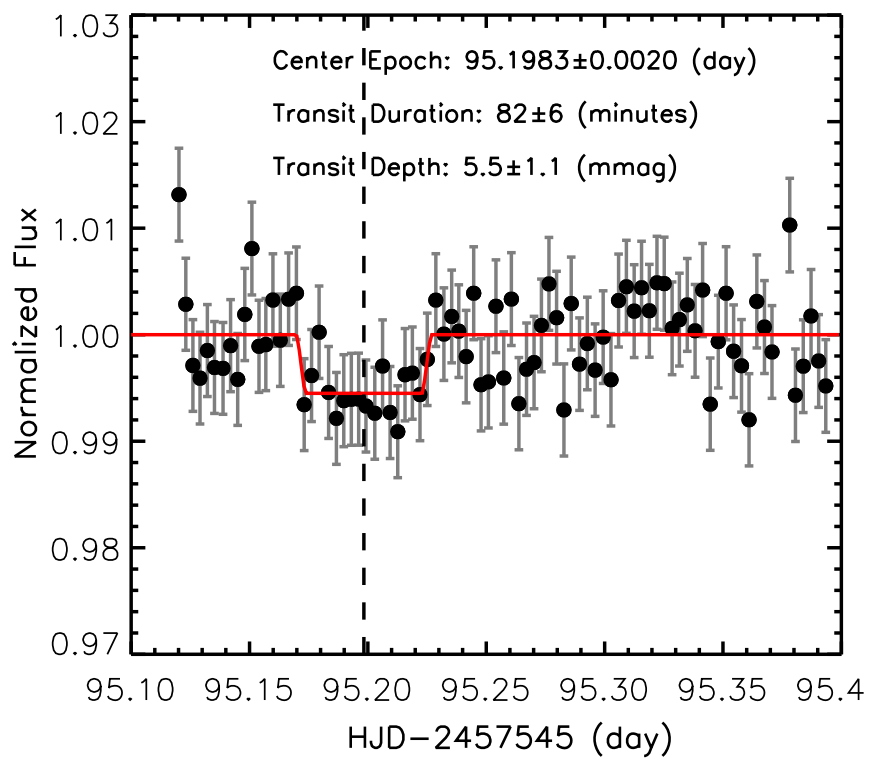

Figure 2. Candidate transit event in the detrended light curve. It is in the $1 \sigma$ window of interest of $T_{\mathrm{IC}}=2,457,640.74 \pm 0.67 \mathrm{HJD}$ for the 86th epoch. The best-fitted model, with $T_{\mathrm{C}}=2,457,640.1983 \pm 0.0020 \mathrm{HJD}, T_{d}=82 \pm 6$ minutes, and $D=5.5 \pm 1.1 \mathrm{mmag}$, is overplotted (the red solid curve).

The variance of $F_{j}$ is then considered as the time-correlated noise, yielding $\sigma_{\mathrm{tc}}=2.5 \mathrm{mmag} .{ }^{13}$ The statistical significance for the candidate transit detection is therefore $S_{\mathrm{tc}}=D / \sigma_{\mathrm{tc}}=$ $5.5 / 2.5=2.2$, where $D=5.5 \mathrm{mmag}$ is the transit depth obtained in Section 4.1.

\subsection{A Gaussian Process Framework}

Time-correlated noise in astronomical data usually has a power spectral density varying as $f^{-\alpha}$ (Collier \& Peterson 2001; Carter \& Winn 2009). In the scheme of Pont et al. (2006), the noise $\sigma_{\text {tc }}$ is estimated as the cumulative time-correlated noise over all timescales appearing in the light curve. Thus, $\sigma_{\mathrm{tc}}$ is dominated by fluctuations at long timescales. However, the time-correlated noise at timescales longer than the transit duration (i.e., a typical timescale of 1-3 hr) has little impact on the planetary transit signals. This is because transit signals can be disentangled from the correlated noise at long timescales effectively. Note that the long-term trend is an extreme case of time-correlated noise at long timescales. Moreover, the noise at short timescales has small amplitudes $\sigma_{\text {tc }} \propto f^{-\alpha}$, which hardly affects the detection threshold. Therefore, the blurring of transit signals is mainly attributed to correlated noise at timescales comparable to the transit duration, if time-correlated noise were the dominant noise source.

Gaussian process (GP) models define a distribution over function space, allowing each point in the time series to have some degree of correlation with every other point. GP regression has been used to model the systematics in a nonparametric way for planetary transit and RV observations (e.g., Gibson et al. 2012; Rajpaul et al. 2015; Kipping et al. 2017). Furthermore, GP regression is an interpolation, as well as a

\footnotetext{
$13 \sigma_{\mathrm{tc}}$ consists of white noise, $\sigma_{w}$, and time-correlated noise. In this case, the sliding windows generally have 16 data points. Then, the white noise can be estimated as $\sigma_{w}=\sigma_{s} / \sqrt{16}=1.15 \mathrm{mmag}$, where $\sigma_{s}=4.6 \mathrm{mmag}$ is the average precision of a single photometric datum. Thus, $\sigma_{\text {tc }}$ is mostly contributed by time-correlated noise.
} 


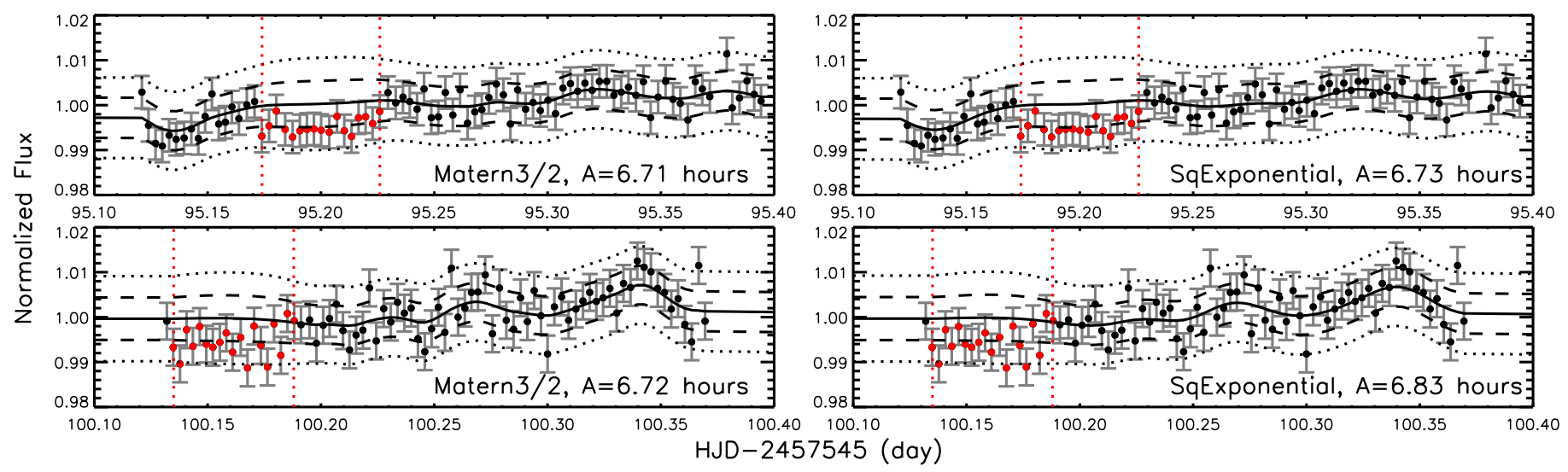

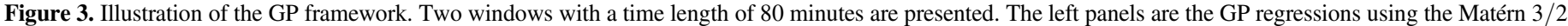

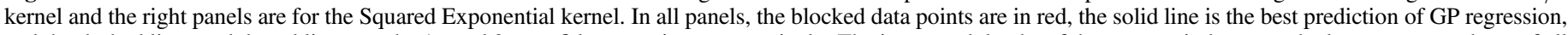

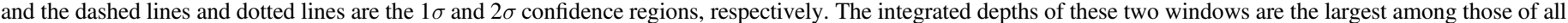

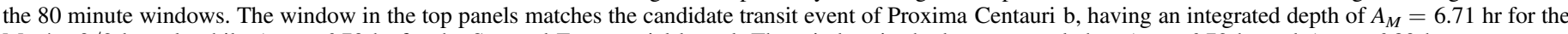
Matérn $3 / 2$ kernel, while $A_{\mathrm{SE}}=6.73 \mathrm{hr}$ for the Squared Exponential kernel. The window in the bottom panels has $A_{M}=6.72 \mathrm{hr}$ and $A_{\mathrm{SE}}=6.83 \mathrm{hr}$.

fitting, technique. It can be used to reconstruct noisy, irregularly sampled data (Rybicki \& Press 1992; Press et al. 2007). In the following, we gauge the significance of the candidate transit event of Proxima Centauri b observed in the BSST light curve in a GP framework.

The full normalized light curve is regressed with GP to model the correlated noise. ${ }^{14}$ Two popular GP kernels, the Matérn 3/2 kernel and Squared Exponential kernel, are adopted for regression. There are three hyperparameters, $\theta_{\text {hyper }}=(\alpha, l, \sigma)$, where $\alpha$ controls the magnitude (in units of mmag), $l$ controls the timescale of correlations (in units of minutes), and $\sigma$ represents the uncertainties of data points (in units of mmag). Regression yields $\theta_{\text {hyper }}=\left(3.31_{-0.32}^{+0.37}, 29.86_{-6.67}^{+8.31}, 4.05_{-0.14}^{+0.15}\right)$ for the Matérn $3 / 2$ kernel and $\theta_{\text {hyper }}=\left(3.20_{-0.32}^{+0.34}, 23.54_{-3.67}^{+4.24}, 4.11_{-0.13}^{+0.13}\right)$ for the Squared Exponential kernel.

We then interrupt the full light curve by blocking data points in sliding windows with varying lengths. Thus, the interrupted light curves have different midtimes of blocked windows and different window lengths from each other. For a specific window length, over 500 interrupted light curves are produced. We perform GP regression as an interpolation to predict the flux at the original time stamps in the blocked windows of the light curves, while the hyperparameters are fixed to those obtained in the full light curve. The predicted flux is considered as the best linear unbiased prediction of the intermediate flux under the priors of the GP model fit to the light curve (Press et al. 2007). We define an integrated depth $A=\int_{t_{0}}^{t_{1}}\left(F_{\text {pre }}-F_{\text {obs }}\right) d t$, where $t_{0}$ and $t_{1}$ are the edges of the blocked windows, $F_{\text {pre }}$ is the predicted flux, and $F_{\text {obs }}$ is the observed flux. For the interrupted light curves with the same window length, the variation of $A$ quantifies the fluctuation induced by correlated noise at timescales comparable to the window length. Although correlated noise at shorter timescales and the white noise contribute to the variation of $A$ as well, their amplitudes are smaller.

In order to illustrate the GP framework, we present two interrupted light curves with blocked windows with length of 80 minutes in Figure 3. Both GP models with the Matérn 3/2

\footnotetext{
${ }^{14}$ We performed GP regression using the Python package developed by Gibson et al. (2012). The package is accessible through https://github.com/ nealegibson/GeePea.
}

kernel and Squared Exponential kernel are presented. The blocked data points are in red. The solid line is the best prediction from GP regression, and the dashed lines and dotted lines are the $1 \sigma$ and $2 \sigma$ confidence regions, respectively. The window in the top panels is the one that matches the candidate transit event of Proxima Centauri b. It has an integrated depth of $A_{M}=6.71 \mathrm{hr}$ for the Matérn $3 / 2 \mathrm{kernel}$, while $A_{\mathrm{SE}}=6.73 \mathrm{hr}$ for the Squared Exponential kernel. The window in the bottom panels has $A_{M}=6.72 \mathrm{hr}$ and $A_{\mathrm{SE}}=6.83 \mathrm{hr}$. It is not associated with possible transits of Proxima Centauri b, since its midtime is far away from the predicted transit epochs. The integrated depths of these two windows are the largest among those of the sliding windows with the same length (80 minutes).

The distribution of the integrated depths for all of the windows with length of 80 minutes is presented in Figure 4. GP regressions using the Matérn $3 / 2$ kernel and Squared Exponential kernel give similar distributions. The integrated depth $A>0$ is attributed to the depression of the observed flux compared to the predicted flux, while $A<0$ is for the excess of the observed flux compared to the predicted flux. The integrated depths distribute more widely in the range of $A<0$. The asymmetry is expected, as Proxima Centauri shows frequent stellar flaring (Davenport et al. 2016), enhancing the probability of flux excess. Thus, only the integrated depths distributed in the range of $A>0$ can be used to gauge the fluctuation purely induced by time-correlated noise. We mirror the integrated depths of $A>0$ to construct a nominal full distribution and report its standard deviation as the fluctuation induced by correlated noise. For the time length of 80 minutes, the standard deviation is $\sigma_{M}=2.66 \mathrm{hr}$ for the GP regressions using the Matérn $3 / 2 \mathrm{kernel}$ and $\sigma_{\mathrm{SE}}=2.67 \mathrm{hr}$ for the Squared Exponential kernel.

The standard deviations of the integrated depths for different window lengths are presented in Figure 5. They increase with window lengths, as the correlated noise with longer timescales have larger amplitudes. We then collect the different-size windows centered at the midtime of the candidate transit event of Proxima Centauri b. Their integrated depths peak at the time lengths close to the transit duration (as seen in Figure 5). In the grid of our GP analysis, the peak integrated depths appear at the window lengths of 76, 78, and 80 minutes. These three windows contains the same photometric data points, thus they 

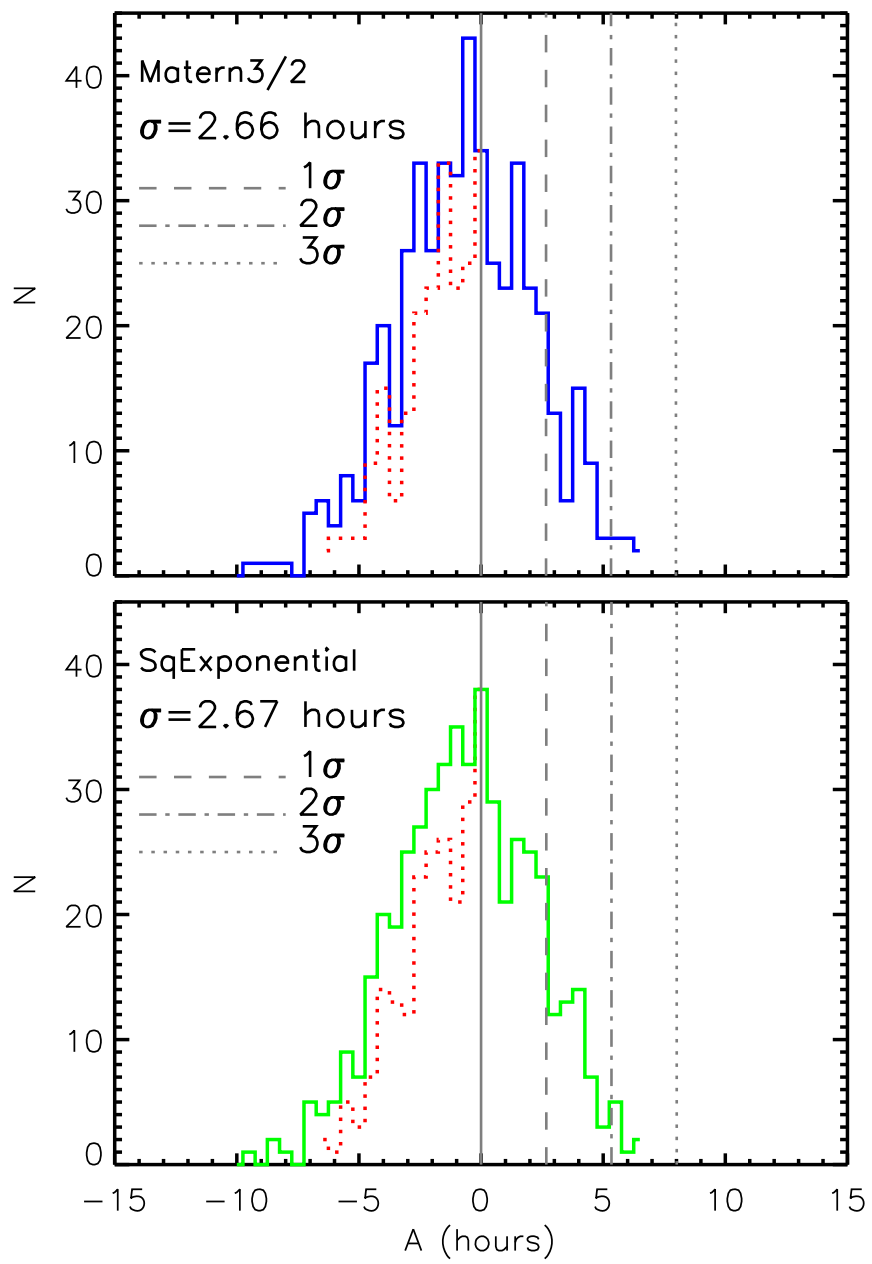

Figure 4. Distribution of integrated depths $A$ for all of the windows with a time length of 80 minutes. GP regressions using the Matern 3/2 kernel (blue) and Squared Exponential kernel (green) give similar distributions. The integrated depths of $A>0$ represent the depression of the observed flux compared to the GP predicted flux, while those with $A<0$ represent the excess. The distribution is asymmetric, as the frequent stellar flaring of Proxima Centauri enhances the probability of flux excess. The integrated depths of $A>0$ are mirrored (red dotted curve) to construct a nominal full distribution. Its standard deviation $\sigma$ is reported as the fluctuation purely induced by time-correlated noise, yielding $\sigma_{M}=2.66 \mathrm{hr}$ (Matérn $3 / 2 \mathrm{GP}$ models) and $\sigma_{\mathrm{SE}}=2.67 \mathrm{hr}$ (Squared Exponential GP models) for the time length of 80 minutes.

are identical. ${ }^{15}$ The window matches the candidate transit event in midtime and length. It is illustrated in Figure 3. Its integrated depth is $A_{M}=6.71 \mathrm{hr}$ for the Matérn $3 / 2 \mathrm{GP}$ model, while $A_{\mathrm{SE}}=6.73 \mathrm{hr}$ for the Squared Exponential GP model. In Figure 5, the standard deviations read $\sigma_{M}=2.52 \mathrm{hr}$ and $\sigma_{\mathrm{SE}}=2.54 \mathrm{hr}$ at the window length of 76 minutes, $\sigma_{M}=$ $2.57 \mathrm{hr}$ and $\sigma_{\mathrm{SE}}=2.57 \mathrm{hr}$ at the window length of 78 minutes, and $\sigma_{M}=2.66 \mathrm{hr}$ and $\sigma_{\mathrm{SE}}=2.67 \mathrm{hr}$ at the window length of 80 minutes. It is straightforward to define the statistic $S=A / \sigma$ as the statistical significance of the transit detections. Conservatively, we report the lowest detection statistic $S=A_{\mathrm{SE}} / \sigma_{\mathrm{SE}}=$ $6.73 / 2.67=2.52$, where the integrated depth and standard deviation at the window length of 80 minutes in the Squared Exponential GP models are employed for the candidate transit event in the BSST light curve. Assuming the distribution of integrated depths is Gaussian, we expect to see two other windows with integrated depths statistically comparable to that of

$\overline{15}$ The time resolution is limited by the sampling rate of the light curve.

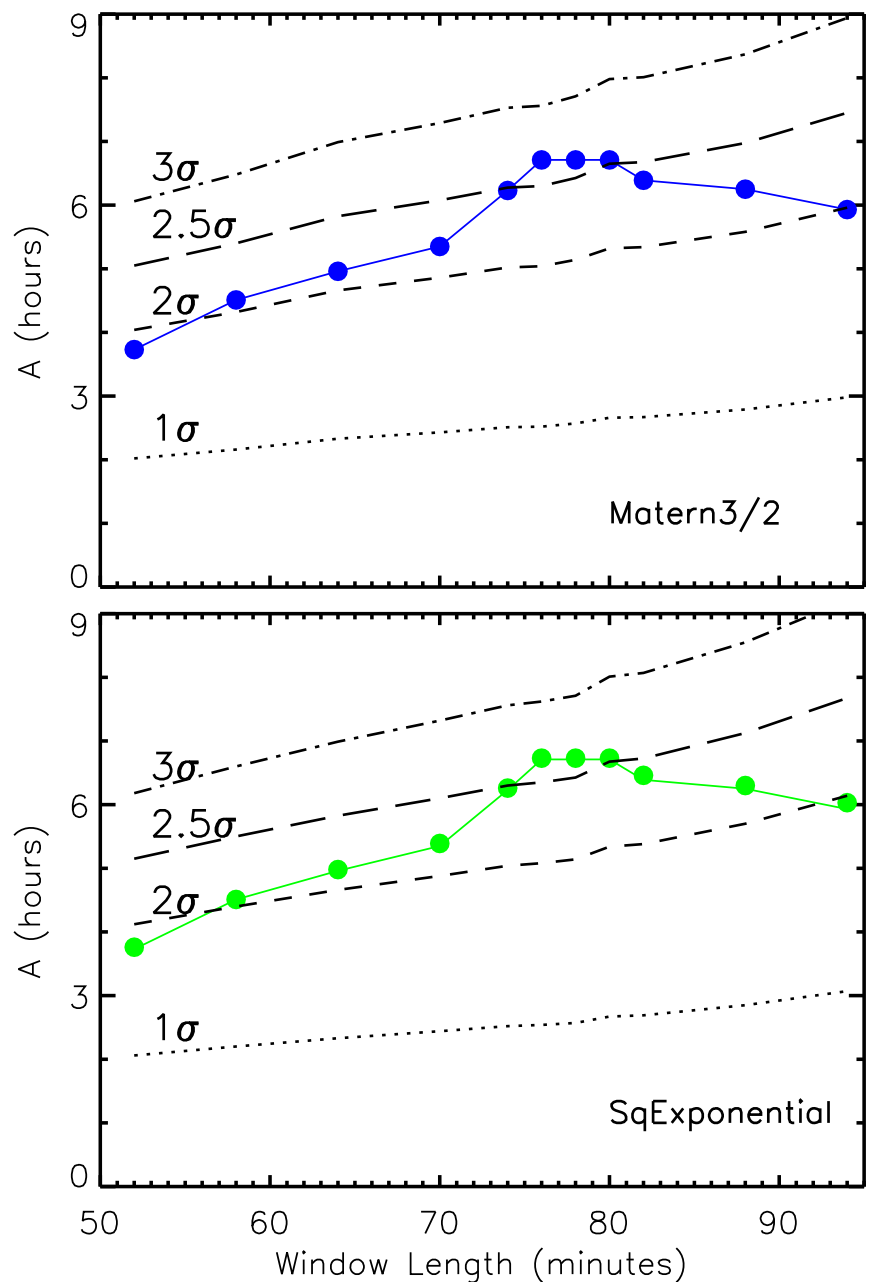

Figure 5. Standard deviations of the integrated depths for different window lengths (dotted and dashed curves) and the integrated depths of different-size windows centered at the midtime of the candidate transit event (color points). The integrated depths peak at the time lengths of 76, 78, and 80 minutes, which are close to the transit duration, in our grid of GP analysis. These three windows contains the same photometric data points, thus they are identical. That window matches the candidate transit event in midtime and length. Its integrated depth is over the $2.5 \sigma$ threshold in both the Matérn 3/2 GP model and Squared Exponential GP model cases.

the tentative transit signal among the 500 windows in the analysis. We do see one as shown in the bottom panels of Figure 3. The candidate transit signal of Proxima Centauri $b$ is identified by the consistency of its midtime and the predicted transit epochs based on the RV solution.

We further normalize the observed light curve using the GP predicted light curve and present the candidate transit event in Figure 6. The best-fitted transit model yields $T_{\mathrm{C}}=$ $2,457,640.1990 \pm 0.0017 \mathrm{HJD}, T_{d}=82.6 \pm 5.3$ minutes, and $D=5.2 \pm 1.0 \mathrm{mmag}$ for GP regression using the Matérn $3 / 2$ kernel, while $T_{\mathrm{C}}=2,457,640.1990 \pm 0.0017 \mathrm{HJD}, \quad T_{d}=$ $82.7 \pm 5.3$ minutes, and $D=5.2 \pm 1.0 \mathrm{mmag}$ for GP regression using the Squared Exponential kernel. Note that the fit is just nominal, as correlated noise is not considered fully. We also attempt to fit the observed flux directly with a full model (Gibson et al. 2012; Kipping et al. 2017), which is a planetary transit model joined with a GP to model the correlated noise. However, the parameters of the transit model are not converged. The divergence is understandable, since the 


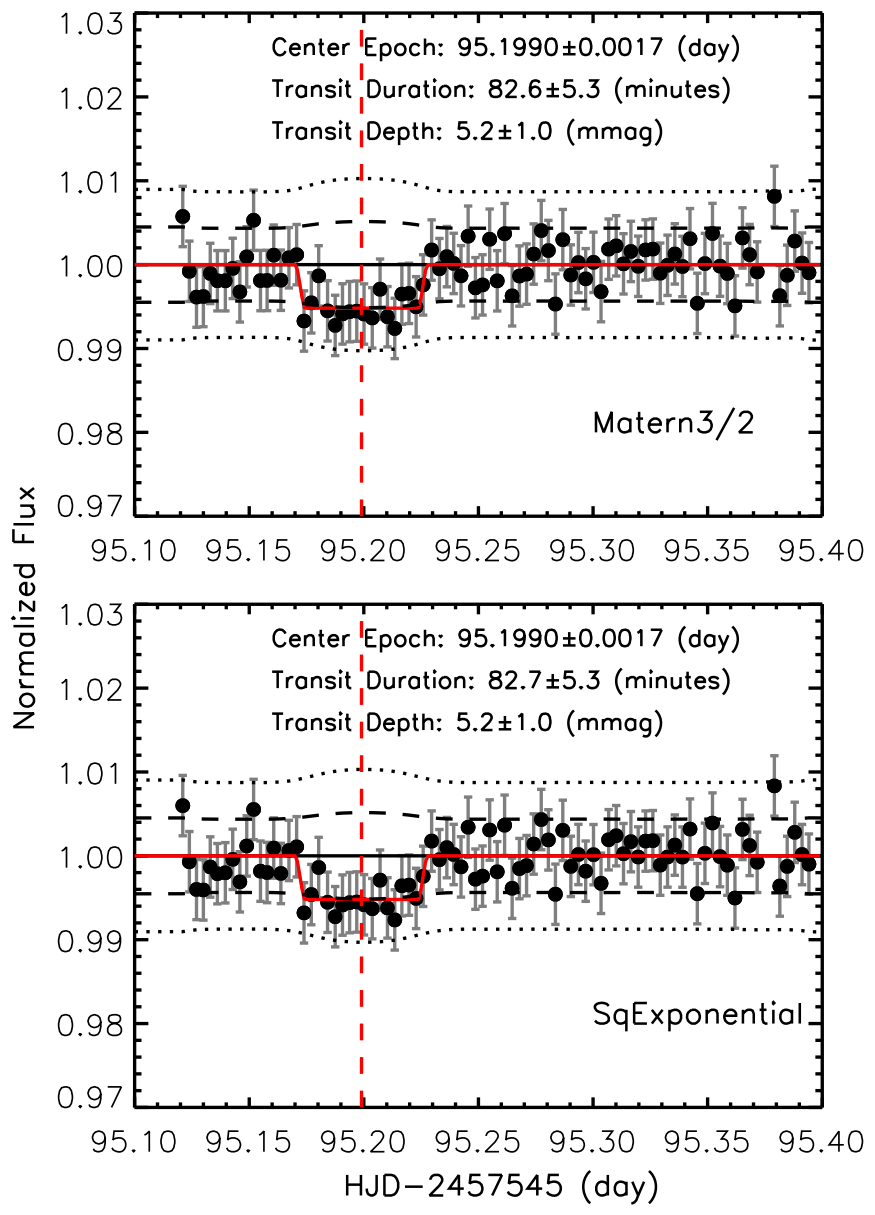

Figure 6. Nominal fit to the candidate transit event of Proxima Centauri b. The observed flux is normalized by the predicted flux from GP regression. The bestfitted transit model (red solid line) yields $T_{\mathrm{C}}=2,457,640.1990 \pm 0.0017 \mathrm{HJD}$ (the vertical red dashed line), $T_{d}=82.6 \pm 5.3$ minutes, and $D=5.2 \pm 1.0$ mmag for GP regression using the Matérn $3 / 2$ kernel, while $T_{\mathrm{C}}=2,457,640.1990 \pm 0.0017 \quad \mathrm{HJD}, \quad T_{d}=82.7 \pm 5.3 \quad$ minutes, and $D=5.2 \pm 1.0 \mathrm{mmag}$ for GP regression using the Squared Exponential kernel. The dashed lines and dotted lines are the $1 \sigma$ and $2 \sigma$ confidence regions of the GP regressions, respectively.

detection of the transit event is just tentative, with a confidence level of $\sim 2.5 \sigma$.

\section{Discussion}

\subsection{Follow-up Observations}

The detection of the transit event of Proxima Centauri $b$, in this work, is tentative, at a confidence level of $2.5 \sigma$. Therefore, four such detections of transit, at least, are required to solidify the transit of Proxima Centauri b at a $5 \sigma$ confidence level. Consecutive high-cadence observations are recommended in order to disentangle reliably the transit signals from correlated noise and frequent stellar flaring. Considering the relatively large uncertainty of the predicted inferior conjunction (Anglada-Escudé et al. 2016; Kipping et al. 2017), the $24 \mathrm{hr}$ before and after the predicted transit epochs are the windows of interest.

The Antarctic Survey Telescopes (ASTs) deployed at Dome A (the Chinese Antarctic Kunlun station) in Antarctica are the best choice for follow-up observation of Proxima Centauri on the ground. The star is continuously observable in
Table 2

Possible Detection of Transit Timing Variations

\begin{tabular}{lccr}
\hline \hline$N_{\text {tr }}$ & $\begin{array}{c}T_{\text {obs }} \\
\text { HJD-2,450,000 (day) }\end{array}$ & $T_{\text {lin }}$ & $\begin{array}{r}\Delta T(\mathrm{C}-\mathrm{O}) \\
\text { minutes }\end{array}$ \\
\hline 11 & $6,801.0594_{-0.0042}^{+0.0053}$ & $6,801.0439$ & $-22.5_{-6.0}^{+7.6}$ \\
43 & $7,159.0514_{-0.046}^{+0.0046}$ & $7,159.0786$ & $+39.3_{-6.9}^{+6.6}$ \\
86 & $7,640.1990_{-0.0017}^{+0.0017}$ & $7,640.1876$ & $-16.8_{-2.4}^{+2.4}$ \\
\hline
\end{tabular}

Note. $T_{\mathrm{obs}}$ is the time of transit minimum assuming all of the candidate events in MOST and BSST light curves are real transits. $T_{\text {obs }}$ is fitted linearly, yielding a period of $P=11.18858$ days. $T_{\text {lin }}$ is the best-fitted linear epoch. $\Delta T$ is the residual of the linear fit, which is considered to be TTVs.

polar nights, and the large clear-sky fraction $(>90 \%)$ at that site can guarantee nearly uninterrupted monitoring. We plan to observe Proxima Centauri in the next winter at Dome A. An observation in four sections, with a length of two days each, is proposed.

\subsection{Comparing with the Candidate Transit Events in MOST Data}

Analyzing the MOST light curve, Kipping et al. (2017) detected two candidate transit events of Proxima Centauri b with the midtransit time of $2,456,801.0594_{-0.0042}^{+0.0053} \mathrm{HJD}$ (for the 11th epoch) and 2,457,159.0514 ${ }_{-0.0048}^{+0.0046}$ HJD (for the 43rd epoch), with an updated orbital period of $P=11.18725_{-0.00016}^{+0.00012}$ days (see Model 2 in their Table 4). Using this ephemeris, we derive the time of transit minimum for the 86th epoch $t_{\mathrm{IC}}=$ $2,457,640.1032_{-0.0117}^{+0.0098}$ HJD. This is 138 minutes earlier than the candidate transit event detected in BSST data. Although all of the detections are tentative, it is still interesting to discuss the incoherence, which may be of some help in follow-up observations. If all of the candidate transit events of Proxima Centauri $b$ are real, we interpret the misalignment of their epochs due to transit timing variations (TTVs, Agol et al. 2005; Holman \& Murray 2005) induced by an outer planet in this system. We fit the three observed transit epochs linearly to derive an optimal orbital period, yielding $P=11.18858$ days. The residuals are therefore the TTVs, $\left\{-22.5_{-6.0}^{+7.6},+39.3_{-6.9}^{+6.6},-16.8_{-2.4}^{+2.4}\right\}$ minutes for the $\{11$ th $, 43 \mathrm{rd}, 86 \mathrm{th}\}$ epochs, as shown in Table 2 . We adopt half of the peak-to-valley value as the strength of the observed TTVs, i.e., TTV $_{\text {obs }} \sim 30$ minutes for Proxima Centauri b.

Assuming a moderate eccentricity $e=0.1$ of Proxima Centauri b (Xie et al. 2016; Brown 2017), we simulate the strengths of its TTVs perturbed by an outer planet with varying masses and orbital periods in coplanar orbits. The mass and orbital period of the outer planet are constrained by the requirement that the Doppler reflex stellar RV should be $<3 \mathrm{~m} \mathrm{~s}^{-1}$ to keep the planet undetectable in the high-precision observation of Anglada-Escudé et al. (2016). The simulation results are presented in the left panel of Figure 7. Strengths of TTVs are significantly enhanced when the planets are nearly in mean motion resonant (MMR) orbits (Xie 2013, 2014). ${ }^{16}$ An Earth-mass planet in orbit near $2: 1$ or $3: 2$ MMR with Proxima Centauri $b$ is able to produce TTVs $\gtrsim 30$ minutes while keeping its reflex stellar $\mathrm{RV}<3 \mathrm{~m} \mathrm{~s}^{-1}$. Since there are only three tentative transits in this work, further analysis of the

\footnotetext{
${ }^{16}$ Note that the planets of TRAPPIST-1 in near MMR orbits exhibit substantial TTVs (Gillon et al. 2017).
} 

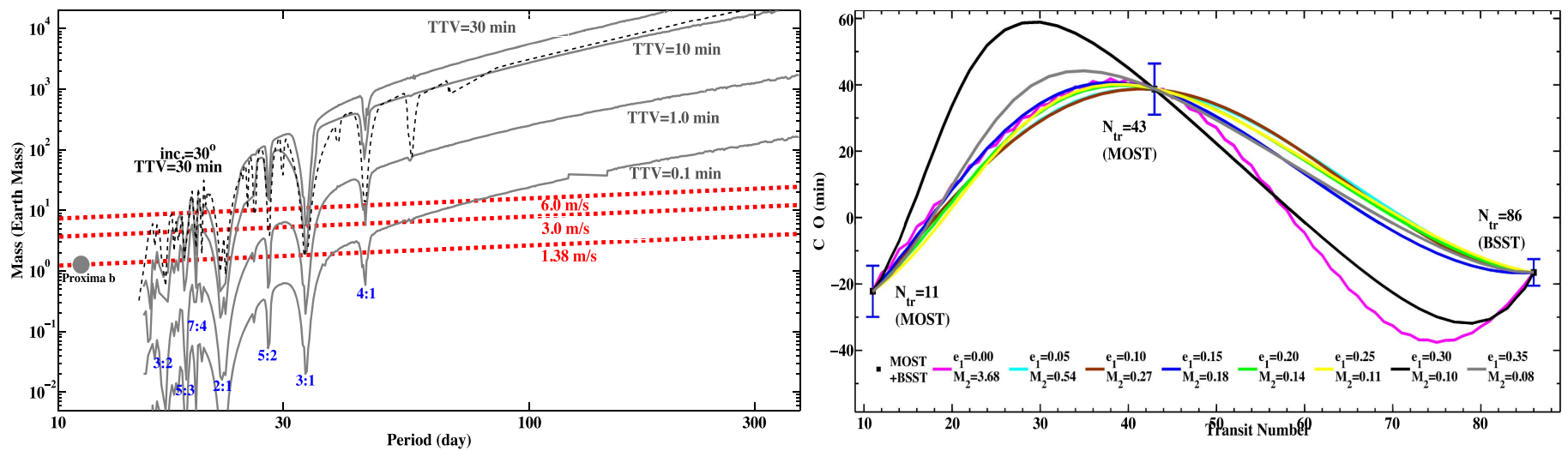

Figure 7. Simulations of TTVs of Proxima Centauri $b$ due to an outer planet. Left panel: TTVs due to an outer planet with various masses and periods. The gray solid lines from top to bottom represent TTV $=30,10,1,0.1$ minutes, respectively. In these simulations, we assume a coplanar orbital configuration of the two planets, where the eccentricity of Proxima Centauri $\mathrm{b}$ is set to $e=0.1$ and the outer planet is in a circular orbit. The black dashed line is a non-coplanar (i.e., inclination $=30^{\circ}$ ) model, producing TTV $=30$ minutes. The red dotted lines represent reflex stellar RV amplitudes induced by the outer planet. Note that any planet with RV amplitude $>3 \mathrm{~m} \mathrm{~s}^{-1}$ can be ruled out by the RV observation of Anglada-Escudé et al. (2016). Right panel: eight arbitrary TTV models fit to the misalignment of the transit epochs observed by MOST and BSST. In these models, the outer planet is in a coplanar near 2:1 mean motion resonance orbit with Proxima Centauri b, and its period is set to 22.58509 days arbitrarily. The curves with different colors represent best-fitted models for different designated eccentricities of Proxima Centauri b, varying from $e=0$ to $e=0.35$ in a step sizes of 0.05 . The best-fitted masses of the outer planet are listed in the legend.

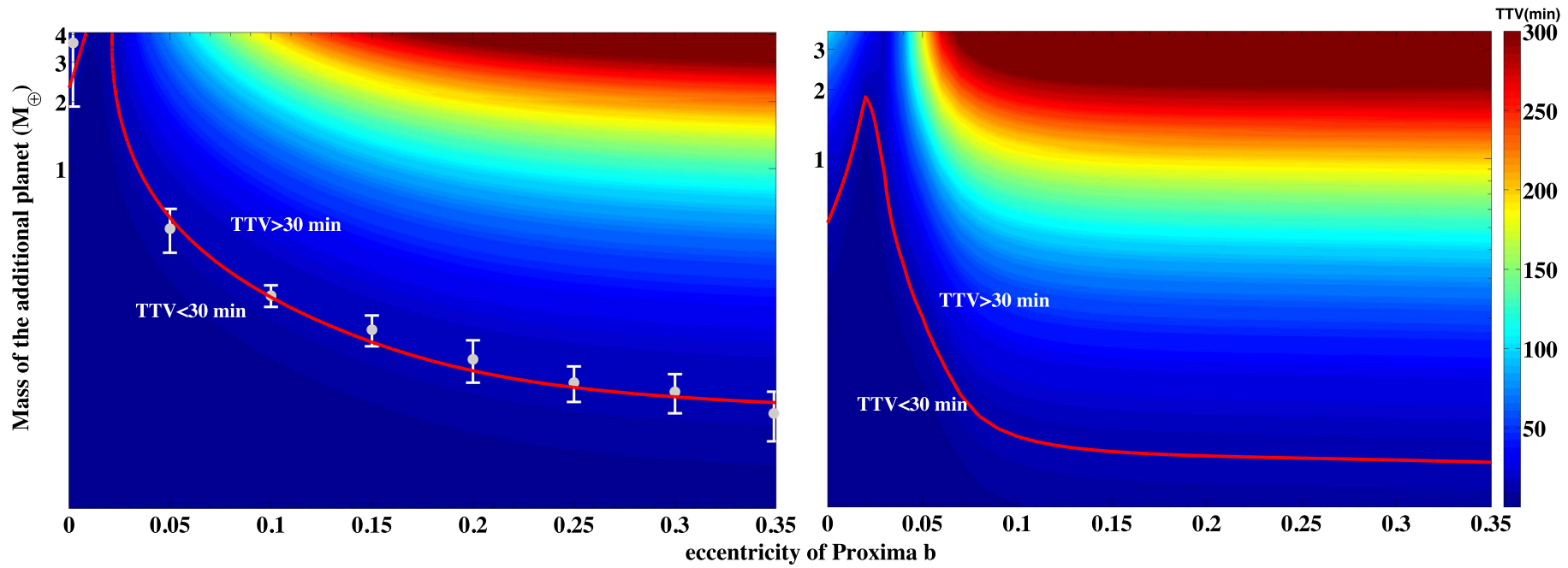

Figure 8. Strengths of simulated TTVs of Proxima Centauri b varying with its eccentricity and the mass of the unseen outer planet, in coplanar near 2:1 and 3:2 MMR orbital configurations. The ranges of the eccentricity and the mass are contained by the RV observation of Anglada-Escudé et al. (2016). The upper limit of the unseen planet is $4.08 M_{\oplus}$ and 3.60 $M_{\oplus}$ for near 2:1 and 3:2 MMR orbital configurations, respectively. In the left panel, the outer planet has an arbitrary orbital period of 22.58509 days, which is in near 2:1 MMR with Proxima Centauri b. In the simulations, the eccentricity of the outer planet is set to zero. The red solid curve represents the TTVs in strength of 30 minutes. The eight filled circles with error bars represent the arbitrary TTV models in the right panel of Figure 7 . The right panel is for the coplanar near 3:2 MMR orbital configuration, where the orbital period of the outer planet is set to 16.89480 days arbitrarily.

mass and orbital elements of the unseen planet, by fitting the observed TTV pattern, is nearly impractical. The situation is worse in this case, as the eccentricity of Proxima Centauri $b$ is quite uncertain $(e<0.35$; Anglada-Escudé et al. 2016) either. However, it is worth constructing some arbitrary TTV models to fit to the misalignment of the transit epochs to illustrate the feasibility of the TTV scenario. The outer planets in the arbitrary models, having an orbital period of 22.58509 days, are coplanar near 2:1 MMR orbits with Proxima Centauri b. Each model has a different designated eccentricity of Proxima Centauri b. In the fitting procedure, we constrain the eccentricity of the outer planet to be small $(<0.05)$, while its orbital phase difference with Proxima Centauri $b$ is a free parameter. The best-fitted models for different designated eccentricities are then presented in the right panel of Figure 7. Generally, the best-fitted mass of the outer planet decreases while the eccentricity of Proxima Centauri b increases. The relationship could be explained by the TTV strength increasing with the eccentricity of Proxima Centauri b when the mass and orbit of the outer planet are set.

Furthermore, we look over the TTV strength varying with the eccentricity of Proxima Centauri $b$ in near 2:1 and 3:2 MMR orbital configurations. First, we simulate TTVs of Proxima Centauri $b$, where the outer planet is in a coplanar near 2:1 MMR orbit. We still use the arbitrary orbital period of 22.58509 days for the outer planet, and set its eccentricity to zero. The mass of the planet in the outer orbit should be less than $4.08 M_{\oplus}$ as the RV constraint (Anglada-Escudé et al. 2016). We present the strengths of simulated TTVs in the left panel of Figure 8. For any specific TTV strength, the required mass of the outer planet decreases with the increasing eccentricity of Proxima Centauri $\mathrm{b}$ in the range of $e \gtrsim 0.02$, 
but a reverse trend is observed in the range of $e \lesssim 0.02$. The turning point can be explained by the two planets being in exact MMR when the eccentricity of Proxima Centauri b is extremely small, and thus the TTV strengths in the range of $e \lesssim 0.02$ are mainly governed by resonant perturbations. In the range of $e \gtrsim 0.02$, the two planets are not in exact MMR, but in near MMR. Therefore, different mechanisms work in the two eccentricity regions. The turning point is more visible in the simulations for the near 3:2 MMR orbital configuration, which are presented in the right panel of Figure 8. There, the unseen planet has an arbitrary orbital period of 16.89480 days and an upper limit mass of $3.60 M_{\oplus}$.

Our brief discussion on TTVs aims to demonstrate that the misalignment of the three tentative transit events can be reasonably interpreted as TTVs of Proxima Centauri b induced by an outer unseen planet. The determination of the mass and orbit of that planet is impractical in this case and therefore beyond the scope of this work.

\section{Summary}

Proxima Centauri is the nearest star to the Sun, with a distance of about $1.3 \mathrm{pc}$. An Earth-mass planet with an orbital period of $\sim 11$ days around it has been revealed by highprecision RV observations. It is interesting that the surface of Proxima Centauri $\mathrm{b}$ is probably temperate and habitable. The geometric probability of transit is about $1.5 \%$. If the planet transits, its radius, atmospheric properties, and habitability can be well studied. We collected high-cadence light curves of Proxima Centauri in 2016 August and September using the BSST at the Zhongshan Station in Antarctica. We detected a tentative transit event at the epoch of $\sim 2,457,640.2$ HJD, which is compatible with the ephemeris of the RV orbit. Timecorrelated noise is pronounced in the light curve of Proxima Centauri, while correlated noise at timescales comparable to the transit duration affect the detection of transit events. We develop a technique, in a Gaussian process framework, to gauge the statistical significance of the candidate transit event in this work, yielding a confidence level of $2.5 \sigma$. Consecutive high-cadence observations are necessary to confirm the planetary transit of Proxima Centauri b. Infrared photometry is preferred, as the stellar noise would be much weaker in that band. Considering the relatively large uncertainty of the predicted inferior conjunction and possible TTVs, we recommend extending the observing windows by one day before and after the predicted epochs. We plan to perform follow-up observation of Proxima Centauri in the next polar night at Dome A in Antarctica, where Proxima Centauri is observable all day.

The authors appreciate the enlightening suggestions from the anonymous referee, which helped to greatly improve the quality of this paper. This work is supported by the Astronomical Project for the Chinese Antarctic Inland Station, the SOC Program (CHINARE2012-02-03, CHINARE2013-02-03, CHINARE201402-03, CHINARE2015-02-03, and CHINARE2016-02-03), and the National Basic Research Program of China (2013CB834905, 2013CB834900, and 2015CB857005). H.-G.L. is supported by the National Natural Science Foundation of China (11503009, 11333002). P.J. is supported by the National Natural Science Foundation of China (11233002, U1431229). X.H. acknowledges support from the China Postdoctoral Science Foundation
(2015M582000). Q.-G.T. is supported by the National Natural Science Foundation of China (11503023), the Natural Science Foundation of Shanghai (14ZR1444100), and the Polar Science Innovation Fund for Young Scientists of Polar Research Institute of China (CX20130201). H.Z. is supported by the National Natural Science Foundation of China (11473025, 11421303, 11033007). The authors express sincere appreciation to $\mathrm{Mr}$. Yongxiang Tang (the leader) and all team members at the Chinese Zhongshan Station, who made the operation of BSST in Antarctica possible in the winter of 2016.

\section{ORCID iDs}

Hui-Gen Liu (1D https://orcid.org/0000-0001-5162-1753

Peng Jiang (1) https://orcid.org/0000-0002-5387-7952

Tuo Ji (iD https://orcid.org/0000-0002-9000-4915

Shaohua Zhang (iD https://orcid.org/0000-0001-8485-2814

Xiheng Shi (i1) https://orcid.org/0000-0002-6710-7537

Ji Wang (1) https://orcid.org/0000-0002-4361-8885

Ji-Lin Zhou (1) https://orcid.org/0000-0003-1680-2940

Hongyan Zhou (iD https://orcid.org/0000-0003-1956-9021

\section{References}

Agol, E., Steffen, J., Sari, R., \& Clarkson, W. 2005, MNRAS, 359, 567 Anglada-Escudé, G., Amado, P. J., Barnes, J., et al. 2016, Natur, 536, 437 Bakos, G. Á., Csubry, Z., Penev, K., et al. 2013, PASP, 125, 154 Bonner, C. S., Ashley, M. C. B., Cui, X., et al. 2010, PASP, 122, 1122 Bouchy, F., Pepe, F., \& Queloz, D. 2001, A\&A, 374, 733

Boyajian, T. S., von Braun, K., van Belle, G., et al. 2012, ApJ, 757, 112 Brown, R. A. 2017, ApJ, 844, 100

Carter, J. A., \& Winn, J. N. 2009, ApJ, 704, 51

Charbonneau, D., Berta, Z. K., Irwin, J., et al. 2009, Natur, 462, 891

Christian, D. J., Mathioudakis, M., Bloomfield, D. S., Dupuis, J., \& Keenan, F. P. 2004, ApJ, 612, 1140

Collier, S., \& Peterson, B. M. 2001, ApJ, 555, 775

Davenport, J. R. A., Kipping, D. M., Sasselov, D., Matthews, J. M., \& Cameron, C. 2016, ApJL, 829, L31

Delfosse, X., Forveille, T., Ségransan, D., et al. 2000, A\&A, 364, 217

Demory, B.-O., Ehrenreich, D., Queloz, D., et al. 2015, MNRAS, 450, 2043

Dravins, D., Lindegren, L., Mezey, E., \& Young, A. T. 1998, PASP, 110, 610

Dressing, C. D., Charbonneau, D., Dumusque, X., et al. 2015, ApJ, 800, 135 Dumusque, X., Pepe, F., Lovis, C., et al. 2012, Natur, 491, 207

Fischer, D. A., Anglada-Escude, G., Arriagada, P., et al. 2016, PASP, 128, 066001

Fortney, J. J., Marley, M. S., \& Barnes, J. W. 2007, ApJ, 659, 1661

Gibson, N. P., Aigrain, S., Roberts, S., et al. 2012, MNRAS, 419, 2683

Gillon, M., Jehin, E., Lederer, S. M., et al. 2016, Natur, 533, 221

Gillon, M., Triaud, A. H. M. J., Demory, B.-O., et al. 2017, Natur, 542, 456

Henry, T. J., Jao, W.-C., Subasavage, J. P., et al. 2006, AJ, 132, 2360

Holman, M. J., \& Murray, N. W. 2005, Sci, 307, 1288

Kervella, P., Mignard, F., Mérand, A., \& Thévenin, F. 2016, A\&A, 594, A107

Kervella, P., Thévenin, F., \& Lovis, C. 2017, A\&A, 598, 7

Kipping, D. M., Cameron, C., Hartman, J. D., et al. 2017, AJ, 153, 93

Kiraga, M., \& Stepien, K. 2007, AcA, 57, 149

Kreidberg, L., \& Loeb, A. 2016, ApJL, 832, L12

Kubánek, P., Jelínek, M., French, J., et al. 2008, Proc. SPIE, 7019, 70192S

Kubánek, P., Jelínek, M., Vítek, S., et al. 2006, Proc. SPIE, 6274, 62741V

Law, N. M., Carlberg, R., Salbi, P., et al. 2013, AJ, 145, 58

Li, Z. Y., Lu, H. P., \& Yuan, X. Y. 2015, ChOpL, 13, 11

Mandel, K., \& Agol, E. 2002, ApJL, 580, L171

Meadows, V. S., Arney, G. N., Schwieterman, E. W., et al. 2016, arXiv:1608. 08620

Pepe, F., Lovis, C., Ségransan, D., et al. 2011, A\&A, 534, A58

Pont, F., Zucker, S., \& Queloz, D. 2006, MNRAS, 373, 231

Press, W. H., Teukolsky, S. A., Vetterling, W. T., \& Flannery, B. P. 2007, Numerical Recipes: The Art of Scientific Computing (New York: Cambridge Univ. Press)

Rajpaul, V., Aigrain, S., Osborne, M. A., Reece, S., \& Roberts, S. 2015, MNRAS, 452, 2269 
Rajpaul, V., Aigrain, S., \& Roberts, S. 2016, MNRAS, 456, L6

Ribas, I., Bolmont, E., Selsis, F., et al. 2016, A\&A, 596, A111

Rybicki, G. B., \& Press, W. H. 1992, ApJ, 398, 169

Shi, S.-C., Paine, S., Yao, Q.-J., et al. 2016, NatAs, 1, 0001

Tian, Q. G., Jiang, P., Du, F. J., et al. 2016, Sci. Bull., 61, 383

Turbet, M., Leconte, J., Selsis, F., et al. 2016, A\&A, 596, A112

Wang, S., Zhang, H., Zhou, J.-L., et al. 2014, ApJS, 211, 26
Xie, J.-W. 2013, ApJS, 208, 22

Xie, J.-W. 2014, ApJS, 210, 25

Xie, J.-W., Dong, S., Zhu, Z., et al. 2016, PNAS, 113, 11431

Yang, H., Allen, G., Ashley, M. C. B., et al. 2009, PASP, 121, 174

Yuan, X., Cui, X., Liu, G., et al. 2008, Proc. SPIE, 7012, 152

Zhang, G. Y., Wang, J., Tang, P. Y., et al. 2016, MNRAS, 455, 1654

Zou, H., Zhou, X., Jiang, Z., et al. 2010, AJ, 140, 602 